\title{
FURTHER REMARKS ON A PROBLEM OF MOSER AND A CONJECTURE OF MAWHIN
}

\author{
JAN ANDRES
}

Dedicated to Louis Nirenberg on the occasion of his 70th birthday

Multiplicity results for both periodic and anti-periodic forced oscillations for the pendulum equation are given on the background of a detailed exposition of the results related to the so-called problem of Moser and to the Mawhin conjecture.

\section{Introduction (Phenomenology of the forced pendulum)}

The purpose of this paper is to extend some observations in [3] concerning the pendulum equation

$$
x^{\prime \prime}+a x^{\prime}+b \sin x=p(t),
$$

where $a$ and $b$ are real constants and the forcing term $p$ is mostly assumed continuous $T$-periodic with zero mean value, i.e.

$$
p(t+T) \equiv p(t), \quad \frac{1}{T} \int_{0}^{T} p(t) d t=0 .
$$

The main result presented here, Corollary 1 (as a consequence of Theorem 1), deals with the number of harmonics with an arbitrarily large period $T$ in the case of a sufficiently large damping constant $a$. All of this is related in some way to the problems of J. Moser and J. Mawhin recalled for convenience below (for details and appropriate references see [3]).

1991 Mathematics Subject Classification. 34C25, 34D05, 58F22.

Supported by the grant no. 201-93-2311 of the Grant Agency of Czech Republic. 
MaWhin's Conjecture. Under the above assumptions, equation (1) admits a $T$-periodic solution for any positive value of $a$.

Moser's Problem. (a) The boundedness (on the positive half-line) of the derivatives to all solutions of (1), when $a=0$.

(b) The boundedness of all solutions of (1) as well as their derivatives, when $a>0$.

These two problems are linked through the well-known Massera transformation theorem (see [3] again) which implies that a negative answer to Mawhin's conjecture excludes case (b) of Moser's problem under the same assumptions, while, if case (b) is satisfied, then the conjecture has a positive answer only in a special situation. Observe that, in the frictionless case $(a=0)$ related to (a), equation (1) admits (see e.g. [16], [27], [33], [35]) two geometrically distinct harmonics with an arbitrarily large period $T$. This fact was the starting point for $\mathrm{J}$. Mawhin to formulate his conjecture and is also the main stimulation for us to prove the above mentioned multiplicity result.

Mawhin's conjecture was answered negatively by R. Ortega [30], who has shown the following.

Ortega's Counterexample. For $T \geq 2 \pi a>0$, there exists $p(t) \in$ $C^{\infty}\left(\mathbb{R}^{1}\right)$, T-periodic, and a constant $\gamma_{0}>0$ such that, for all $\gamma>\gamma_{0}$, the problem $x^{\prime \prime}+a \gamma x^{\prime}+\gamma \sin x=\gamma p(t), x(0)-x(T)=x^{\prime}(0)-x^{\prime}(T)=0$ has no solution.

So we tried to reformulate Mawhin's conjecture (see [3]) as follows:

Modified Mawhin's Conjecture. Assume that the $T$-periodic function $p$ has zero mean value. Then, for any $a>0$, the equation (1) has a $T$-periodic solution of second kind, i.e. a solution such that $x(t+T)=x(t)+2 k \pi$ for some $k \in \mathbb{Z}, x^{\prime}(t+T)=x^{\prime}(t)$.

This way seemed quite promising because of many supporting arguments partly presented in [3]. Nevertheless, R. Ortega informed us in a personal letter that the so modified conjecture is false in general. In fact, his counterexample in [30] (with $T=2 \pi a$ ) still works. On the other hand, if $x$ is a $T$-periodic solution of second kind satisfying

$$
x(t+T)=x(t)+2 k \pi \quad \text { for some } k \in \mathbb{Z}, k \neq 0,
$$

then, integrating (1) (with $b=1$ ) from 0 to $T$, we obtain

$$
2 k \pi a+\int_{0}^{T} \sin x(t) d t=0 .
$$

Since $\left|\int_{0}^{T} \sin x(t) d t\right|<T$ (observe that $x(t) \not \equiv \pi / 2+k \pi$ ), we have

$$
2 \pi a \leq|2 k \pi a|<T .
$$


So, the problem of the existence of such a solution leads to considering equation (1) in the weakly dissipative case (i.e. for $a>0$ small).

As we were informed by A. Chenciner (who probably was the first to realize this possibility; see also [10]), there exists an appropriate fixed-point technique (a modification of the Poincaré-Birkhoff fixed-point theorem) for studying weakly dissipative twist maps (see e.g. [7]). This technique has been mainly developed by P. Le Calvez (see e.g. [20], [21] and the references therein) and by M. Casdagli ([8], [9]). This approach consists in proving the existence of the so-called rotation intervals (intervals of rotation numbers) and to associate with each (rational) number of such intervals a (subharmonic) orbit having the same rotation number. There are already some applications for (1) in the presence of damping, when the amplitude of $|p(t)|$ is very small (see e.g. [19]) or $T$ is small enough (see the example in [9] commented in [20]). The results are in a good agreement with the note in [12] deduced by a completely different approach. In the remaining cases the problem seems to be difficult (see $[15])$.

In the absence of damping, the situation is much easier and, what is interesting, it is strictly related through the Birkhoff theorem (see e.g. [7]) to the problem of Moser in the case (a). In this context various results have been obtained recently in [11], [24], [29], [34] (for the details see also [25], [26]), as a by-product of more general results.

F. Zanolin, jointly with T. Ding, has proved a slightly better analogy of the existence result of M. Levi [24] on subharmonics in the frictionless case. Because of the similarity they did not publish it. This problem is in fact of a variational nature as pointed out in [6], where the connection with a certain semilinear parabolic partial differential equation is indicated.

The following rather surprising result has been obtained independently in [22] and [28]: for $a>2 \sqrt{b}$ (and for $p$ with a not necessarily zero mean value) equation (1) has a unique globally attracting invariant circle $(\bmod 2 \pi)$ in the $\left(x, x^{\prime}+a x\right)$ phase-plane. This implies that only nonchaotic motions can appear, because every trajectory is asymptotically periodic or quasi-periodic in the $\left(x, x^{\prime}+a x\right)$ plane.

As pointed out in [22] (see the references therein), chaos has been found in (1), where $b=1$, for $a \approx 1.576$. This shows that $a>2$ is a not too crude estimate for nonchaotic behaviour in (1). The value $a \approx 2 \sqrt{b}$ corresponds (see $[22],[28])$ to the critically damped pendulum, and is in a good agreement with the Lyapunov stability investigation of periodic orbits in [31], where exactly the same value appears. For the underdamped pendulum, $a<2 \sqrt{b}$, the Lagrange stability of (1) can be observed (see [1], [3], [5]) in spite of the chance of a possible chaotic motion inside (see [22], [28]). 
In the overdamping case, $a>2 \sqrt{b}$, the structure of the phase-plane $\left(x, x^{\prime}+\right.$ $a x)$ has been made precise in [23], where (1) is shown to possess an invariant foliation transversal to the invariant attracting circle. An explicit set of additional conditions has been derived for it. An extremely detailed analysis of the corresponding phase-plane to (1) in the weakly dissipative (underdamping) case has been made by various methods in [19], provided the maximal amplitude of $|p(t)|$ is sufficiently small. From our point of view here, especially the structure of the so-called Birkhoff attractors (cf. [7]) is of particular interest.

\section{Multiplicity results}

It is well known (see e.g. [16], [27], [33], [35]) that equation (1) has for $a=0$ at least two geometrically distinct harmonics, provided a $T$-periodic $p$ has a zero mean value. In [33], exactly two harmonics are even guaranteed under the additional restrictions, $b<4 \pi^{2} / T^{2}$ and another explicit condition holding for sufficiently small values of both the maximal amplitude of $|p(t)|$ and the period T. A similar exact result is given in [33] for (1), where $a>0$ is a sufficiently large value proportional to both the maximal amplitude of $|p(t)|$ and $T$ again. As an unpleasant consequence, the criterion in [33] loses its effectiveness for very large $T$.

For the most part, other multiplicity results for $a>0$ have explicitly or implicitly the same defect (see [27]). Checking the literature, we have found only two recent multiplicity criteria not depending on the length of $T$. One of them is a very general multiplicity criterion in [27] (generalizing and refining an earlier one due to H. W. Knobloch), namely

$$
b>\max _{t \in[0, T]}|p(t)|=: P \quad\left(\frac{1}{T} \int_{0}^{T} p(t) d t=0\right) .
$$

This sole condition ensures the existence of at least two $T$-periodic solutions of (1). Another condition without the mentioned defect is

$$
a>2 \sqrt{b}
$$

obtained in [31] under further information about the Lyapunov stability and instability of at least two (but a finite number of) harmonics.

Therefore, in analogy to the frictionless case $(a=0)$, we prove in this section the existence of at least two geometrically distinct $T$-periodic solutions of (1), where $T>0$ can be arbitrarily large.

As will be seen, the conditions obtained in this paper are comparable with those mentioned above, as shown in the example at the end of this section.

It is proved in [5] (see also [1], [3]) that there exists a $T$-periodic solution $x_{1}$ with $x_{1}(\hat{t})=0$ for some $\widehat{t} \in[0, T]$, provided $a>\sqrt{b /(2+\pi)}+\varepsilon(P)$, where $\varepsilon(P) \rightarrow 0$ as $P \rightarrow 0$. Our aim below is to prove the existence of a second solution 
$x_{2}$ satisfying $\left|x_{2}(t)-\pi\right|<\pi$ for all $t$. This will be done under some stronger condition.

Hence, consider equation (1), where $a, b$ are positive constants, $p$ is continuous and

$$
p(t+T) \equiv p(t), \quad \frac{1}{T} \int_{0}^{T} p(t) d t=0 .
$$

Consider the auxiliary equation

$$
x^{\prime \prime}+a x^{\prime}+b \sin _{\delta}^{*} x=p(t),
$$

where

with $0<\delta<\pi$.

$$
\sin _{\delta}^{*} x:= \begin{cases}\sin (x+\pi) & \text { for }|x| \leq \pi-\delta \\ -\sin \delta \operatorname{sgn} x & \text { for }|x| \geq \pi-\delta\end{cases}
$$

Clearly $x_{2}$ is a $T$-periodic solution of (1) satisfying $\left|x_{2}(t)-\pi\right|<\pi$ for all $t$ iff there exists $0<\delta<\pi$ such that $x^{*}=x_{2}-\pi$ is a $T$-periodic solution of $\left(1^{*}\right)$ satisfying $\left|x^{*}(t)\right| \leq \pi-\delta$ for all $t \in \mathbb{R}$.

Our approach is based on the application of the Lemma below elaborated in [4] and further developed in [2].

REMARK 1. $V_{\left(1^{*}\right)}^{\prime}$ and $W_{\left(1^{*}\right)}^{\prime}$ denote the derivatives of $t \mapsto V(x(t))$ and $t \mapsto$ $W\left(t, x(t), x^{\prime}(t)\right)$ respectively, where $x(\cdot)$ is any solution of equation $\left(1^{*}\right)$.

LEMMA. Let a smooth Lyapunov function $V\left(x^{\prime}\right)$ exist such that

(i) $\lim _{\left|x^{\prime}\right| \rightarrow \infty} V\left(x^{\prime}\right)=\infty$,

(ii) there exists $D_{1}>0$ such that $V_{\left(1^{*}\right)}^{\prime}\left(x^{\prime}\right)<0$ for $\left|x^{\prime}\right|>D_{1}$.

Let $W\left(t, x, x^{\prime}\right)$ be a T-periodic (in $\left.t\right)$ smooth Lyapunov function such that

(iii) $\lim _{|x| \rightarrow \infty} W\left(t, x, x^{\prime}\right)=\infty$ for $\left|x^{\prime}\right| \leq \bar{D}_{1}$ uniformly in $t \geq 0$, where $\bar{D}_{1}\left(\geq D_{1}\right)$ may be large,

(iv) there exist $D$ and $S>0$ such that $W_{\left(1^{*}\right)}^{\prime}\left(t, x, x^{\prime}\right) \geq S$ for $|x| \geq D$ and $\left|x^{\prime}\right| \leq \bar{D}_{1}$, uniformly in $t \geq 0$.

Then equation $\left(1^{*}\right)$ admits a T-periodic solution.

THEOREM 1. Assume that

$$
P_{0} / a+\Delta \leq \pi,
$$

where

$$
\begin{aligned}
\Delta & :=\frac{1}{a^{2}}\left\{\Delta_{0}+\left[\Delta_{0}^{2}+3(b+P)^{2}+6 a P_{0}(b+P)+5 a^{2} P^{2}\right]^{1 / 2}\right\}, \\
\Delta_{0} & :=P+2 b+a P_{0}, \quad P:=\max _{t \in[0, T]}|p(t)|, \\
P_{0} & :=\min _{t_{1} \in[0, T]}\left[\max _{t_{2} \in[0, T]}\left|\int_{t_{1}}^{t_{2}} p(t) d t\right|\right]=\max _{t \in[0, T]}\left|\int_{t_{0}}^{t} p(s) d s\right|,
\end{aligned}
$$


where $t_{0}$ denotes the value of $t_{1}$ for which the minimum is attained. Then equation $\left(1^{*}\right)$ admits a T-periodic solution $x$ such that $|x(t)|<\pi$.

Proof. First, we show that $\left(1^{*}\right)$ has a harmonic for any positive value of $a$, i.e. not necessarily for (2).

Defining $V\left(x^{\prime}\right)=x^{\prime 2}$, one can readily check that (i) holds and (ii) is satisfied for $D_{1}=(b+P) / a$. Taking

$$
2 W\left(t, x, x^{\prime}\right)=2 b \int_{0}^{x} \sin _{\delta}^{*} s d s+\left[x^{\prime}+a x-\int_{t_{0}}^{t} p(s) d s\right]^{2}
$$

we have

$$
W_{\left(1^{*}\right)}^{\prime}\left(t, x, x^{\prime}\right)=-b \sin _{\delta}^{*} x\left[a x-\int_{t_{0}}^{t} p(s) d s\right] \geq b\left|\sin _{\delta}^{*} x\right|\left(a|x|-P_{0}\right) .
$$

Therefore, because of the boundedness of $\sin _{\delta}^{*} x$ and $\int_{t_{0}}^{t} p(s) d s$, (iii) is immediately satisfied as well, and (iv) holds for $D>P_{0} / a$. Consequently, the above Lemma applies.

In order to prove that the desired harmonic $x$ can be absolutely estimated by $\pi$, we show that if there exists $t^{*} \geq 0$ such that $\left|x\left(t^{*}\right)\right| \geq \pi$, then

$$
\lim _{t \rightarrow \infty}|x(t)|=\infty
$$

Indeed, let $x$ be such a $T$-periodic solution. It is easy to see (cf. [1], [5]) that

$$
\left|x^{\prime}(t)\right| \leq \frac{b+P}{a}=: D_{1}\left(=: \bar{D}_{1}\right) .
$$

Moreover, we have

(4) $\quad W_{\left(1^{*}\right)}^{\prime}\left(t, x(t), x^{\prime}(t)\right) \geq b\left|\sin _{\delta}^{*} x(t)\right|\left(a|x(t)|-P_{0}\right) \geq a b \varepsilon \sin \delta=: S(>0)$

provided $|x(t)| \geq P_{0} / a+\varepsilon$, where $\varepsilon$ is a positive number. Thus, $W\left(t, x(t), x^{\prime}(t)\right)$ is strictly increasing and

$$
\lim _{t \rightarrow \infty} W\left(t, x(t), x^{\prime}(t)\right)=\infty \quad \text { provided }|x(t)| \geq P_{0} / a+\varepsilon, \forall t \geq t^{*}
$$

Let us write

$$
W\left(t, x, x^{\prime}\right)=W\left(t^{*}+\Delta_{t}, x_{0}+\Delta_{x}, x_{0}^{\prime}+\Delta_{x^{\prime}}\right)=W\left(t^{*}, x_{0}, x_{0}^{\prime}\right)+\Delta_{W},
$$


where

$$
\begin{aligned}
\Delta_{W}= & a x_{0}\left(a \Delta_{x}+\Delta_{x^{\prime}}-\int_{t^{*}}^{t^{*}+\Delta_{t}} p(t) d t\right)+b \int_{x_{0}}^{x_{0}+\Delta_{x}} \sin _{\delta}^{*} x d x+\frac{a^{2}}{2} \Delta_{x}^{2} \\
& +a \Delta_{x}\left(\Delta_{x^{\prime}}-\int_{t_{0}}^{t^{*}+\Delta_{t}} p(t) d t+x_{0}^{\prime}\right)+x_{0}^{\prime} \Delta_{x^{\prime}}+\frac{1}{2} \Delta_{x^{\prime}}^{2} \\
& -x_{0}^{\prime} \int_{t^{*}}^{t^{*}+\Delta_{t}} p(t) d t-\Delta_{x^{\prime}} \int_{t_{0}}^{t^{*}+\Delta_{t}} p(t) d t+\int_{t_{0}}^{t^{*}} p(t) d t \int_{t^{*}}^{t^{*}+\Delta_{t}} p(t) d t \\
& +\frac{1}{2}\left(\int_{t^{*}}^{t^{*}+\Delta_{t}} p(t) d t\right)^{2} .
\end{aligned}
$$

Let us show that $\Delta_{W} \leq 0$. This will lead to a contradiction with (4) whenever $|x(t)| \geq P_{0} / a+\varepsilon$ for all $t \in\left[t^{*}, t^{*}+\Delta_{t}\right]$.

Since (take $\Delta_{x}=-\left|\Delta_{x}\right| \operatorname{sgn} x_{0}$ )

$$
\begin{aligned}
\Delta_{W} \leq & a\left|x_{0}\right|\left(-a\left|\Delta_{x}\right|+P_{0}\right)+\frac{a^{2}}{2} \Delta_{x}^{2}+\left|\Delta_{x}\right|\left(P+2 b+a P_{0}\right) \\
& +|| x_{0}|-| \Delta_{x}||(b+P)+\frac{3}{2} \frac{(b+P)^{2}}{a^{2}}+2 \frac{b+P}{a} P_{0}+\frac{3}{2} P_{0}^{2},
\end{aligned}
$$

the desired inequality, $\Delta_{W} \leq 0$, holds for $\left|x_{0}\right| \geq P_{0} / a+\varepsilon+\left|\Delta_{x}\right|$ whenever (see the definition of $\Delta_{0}$ above and take $\left.\left|x_{0}\right|=P_{0} / a+\varepsilon+\left|\Delta_{x}\right|\right)$

$\frac{a^{2}}{2} \Delta_{x}^{2}+\varepsilon a^{2}\left|\Delta_{x}\right| \geq\left|\Delta_{x}\right| \Delta_{0}+\frac{3}{2} \frac{(b+P)^{2}}{a^{2}}+\left(3 \frac{b+P}{a}+\varepsilon a\right) P_{0}+\frac{5}{2} P_{0}^{2}+\varepsilon(b+P)$,

i.e. when

$$
\begin{aligned}
\left|\Delta_{x}\right| \geq & \frac{1}{a^{2}}\left\{\Delta_{0}+\left[\Delta_{0}^{2}+a^{4} \varepsilon^{2}-2 \varepsilon a^{2}(P+2 b)+3(b+P)^{2}\right.\right. \\
& \left.\left.+6 a P_{0}(b+P)+5 a^{2} P^{2}\right]^{1 / 2}+2 \varepsilon a^{2}(b+P)\right\}-\varepsilon=: \Delta_{\varepsilon} .
\end{aligned}
$$

Set $x_{0}=x\left(t^{*}\right), x_{0}^{\prime}=x^{\prime}\left(t^{*}\right)$ and $\left|\Delta_{x}\right|=\Delta_{\varepsilon}$. So, if $\left|x\left(t^{*}\right)\right|=\left|x_{0}\right| \geq \pi$ $\left(\geq P_{0} / a+\Delta \ldots\right.$, see $\left.(2)\right)$, then one can easily verify that there exists a sufficiently small $\varepsilon>0$ such that $\Delta \geq \varepsilon+\Delta_{\varepsilon}$, i.e. $\left|x_{0}\right| \geq P_{0} / a+\varepsilon+\Delta_{\varepsilon}$, which implies both $\Delta_{W} \leq 0$ and $|x(t)|>P_{0} / a+\varepsilon$ on some interval $\left[t^{*}, t_{1}\right)$.

Since there is no $t_{1}:=t^{*}+\Delta_{t}>t^{*}$ for which $\left|x\left(t_{1}\right)\right|=P_{0} / a+\varepsilon$ (otherwise, we get simultaneously $W\left(t_{1}, x\left(t_{1}\right), x^{\prime}\left(t_{1}\right)\right)>W\left(t^{*}, x\left(t^{*}\right), x^{\prime}\left(t^{*}\right)\right)$ by $(4)$ and $W\left(t_{1}, x\left(t_{1}\right), x^{\prime}\left(t_{1}\right)\right) \leq W\left(t^{*}, x\left(t^{*}\right), x^{\prime}\left(t^{*}\right)\right)$ since $\left.\Delta_{W} \leq 0\right)$, we have $|x(t)|>P_{0} / a+$ $\varepsilon$ for all $t \geq t^{*}$, and consequently $\left(4^{\prime}\right)$ must be satisfied.

Since $W\left(t, x, x^{\prime}\right)$ is bounded in $t$ and $\left|x^{\prime}(t)\right| \leq D_{1}$ for all $t$, condition (3) holds (observe that

$$
W\left(t, x, x^{\prime}\right)=\int_{0}^{x} \frac{\partial W\left(t, x, x^{\prime}\right)}{\partial x} d x+\frac{1}{2}\left[x^{\prime}-\int_{t_{0}}^{t} p(s) d s\right]^{2},
$$

where $\left.\left|\partial W\left(t, x, x^{\prime}\right) / \partial x\right| \leq a^{2}|x|+\left(2 b+P+a P_{0}\right)\right)$, which contradicts the periodicity of $x(t)$. Therefore, $|x(t)|<\pi$, which completes the proof. 
Corollary 1. Under condition (2), where

$$
P_{0}:=\max _{t_{1}, t_{2} \in[0, T]}\left|\int_{t_{1}}^{t_{2}} p(t) d t\right| \quad\left(P:=\max _{t \in[0, T]}|p(t)|\right),
$$

equation (1) has at least two geometrically distinct T-periodic solutions.

ProOF. As indicated above, it directly follows from Theorem 1 that under condition (2) equation (1) admits a $T$-periodic solution $x_{2}$ such that $\left|x_{2}(t)-\pi\right|$ $<\pi$. Similarly, we have proved in [5] (cf. also [1]) that for

$$
a>(b+P) \frac{P_{0}+\left\{P_{0}^{2}+4[2 b+\pi(b+P)]\right\}^{1 / 2}}{2[2 b+\pi(b+P)]},
$$

where $P_{0}$ is as above, there exists another harmonic $x_{1}$ with $x_{1}(\widehat{t})=0$ for some $\widehat{t} \in[0, T]$.

Under the assumptions of Theorem 1 , we can obviously replace the original definition of $P_{0}$ by the stronger one, $P_{0}=\max _{t_{1}, t_{2} \in[0, T]}\left|\int_{t_{1}}^{t_{2}} p(t) d t\right|$ as above. So, one only has to show that the mentioned assertion in [5] is also true with (2) instead of (5).

Since (5) is equivalent (see [5]) to the inequality

$$
a \pi+\frac{2 a b}{b+P}>P_{0}+\frac{b+P}{a},
$$

and (2) can be rewritten as

$$
a \pi \geq P_{0}+\frac{1}{a}\left\{P+2 b+a P_{0}+[\cdot]^{1 / 2}\right\},
$$

the fact that (5) follows from (2) can be verified by a straightforward calculation.

ExAmple. For $b=P=P_{0}$, condition (2) reads

$$
b \leq \frac{\pi a^{2}}{3+4 a+\sqrt{21+22 a+\frac{37}{3} a^{2}}} .
$$

In [31] it is assumed $b<a^{2} / 4$. Observe that if e.g. $a=1 / 2$, then the constant obtained here is $\approx 3 / 44$, which is greater than $3 / 48$, the constant in [31].

REMARK 2. By the substitution $\tau:=-t$, the results of this section remain certainly valid for $a$ replaced in (2) and (5) by $|a|$.

REMARK 3. The above result can be easily generalized to the equation

$$
x^{\prime \prime}+a x^{\prime}+h(x)=p(t),
$$

where the restoring term $h$ is a suitable (not necessarily periodic) alternating function with sufficiently large distances between its zeros. 
REMARK 4. The same idea can be applied in a rather more complicated way to higher-order periodically forced pendulum-type equations. The results will appear elsewhere.

\section{Anti-periodic oscillations}

In many technical experiments, equation (1), where $p(t)$ has a sinusoidal character, plays the role of a paradigm (see e.g. [14], [18], [22], [28] and the references therein). The observations, numerical simulations and measurements are then frequently regarded as typical and comparable to those with other types of forcing torques. Thus, for example, the existence of two $2 \pi$-periodic orbits observed in [18] for the typical "chaotic" equation (cf. [14])

$$
x^{\prime \prime}+0.2 x^{\prime}+\sin x=2 \cos t
$$

may seem encouraging to prove the same also for a different $2 \pi$-periodic forcing term with zero mean value. However, it is not so in general.

The reason is that $\cos t$ is not only $2 \pi$-periodic, but also $\pi$-anti-periodic, which is crucial as we will see below. Although all the criteria known to us (see [3]) fail here when $p$ is only $2 \pi$-periodic, but not $\pi$-anti-periodic, we will show easily that equation (6) admits a $2 \pi$-periodic solution.

Consider the following anti-periodic (half-periodic or skew-periodic) boundary value problem

$$
x^{\prime \prime}+a x^{\prime}+b \sin x=p(t), \quad x(0)=-x(T), x^{\prime}(0)=-x^{\prime}(T),
$$

where again $p \in C([0, T])$.

It is clear that every solution $x$ of (1) is $2 T$-periodic if $p(t+T) \equiv-p(t)$; for example, $p(t):=\cos t$ for $T=\pi$.

In the $C^{2}([0, T])$-class, problem ( 7$)$ is obviously equivalent to

$$
x(t)=\int_{0}^{T} G(t, s)\left[p(s)-b \sin x(s)-a x^{\prime}(s)\right] d s=: F(x(t)),
$$

where

$$
G(t, s)= \begin{cases}\frac{1}{2}(t-s-T / 2) & \text { for } 0 \leq s \leq t \leq T \\ \frac{1}{2}(s-t-T / 2) & \text { for } 0 \leq t \leq s \leq T\end{cases}
$$

Since the operator $F(\cdot)$ is completely continuous (see e.g. [13]), the Schauder fixed-point theorem applies (see [13]), provided there exists a closed convex subset $S$ of the Banach space $B$ of all continuously differentiable functions on $[0, T]$, with the norm

$$
\|x\|:=\max _{t \in[0, T]}\left[|x(t)|+\left|x^{\prime}(t)\right|\right]
$$

such that $F(S) \subset S$. 
Define

$$
S:=\left\{x \in B:\|x\| \leq D, D \in \mathbb{R}^{+}\right\} .
$$

It is clear that $S$ is closed and convex. Thus, it is enough to show that for $x \in S$ we have

$$
\|F(x)\| \leq D
$$

where $D$ is a suitable positive constant. One has

$$
\max _{t \in[0, T]}\left\{\max _{s \in[0, T]}\left[|G(t, s)|+\left|\frac{\partial G(t, s)}{\partial t}\right|\right]\right\} \leq \frac{T}{4}+\frac{1}{2},
$$

where $G$ is the Green function defined above. It can be easily verified that (8) holds for

$$
|a|<\frac{4}{T(T+2)}
$$

Hence, we can finally give

Theorem 2. Assume that the continuous function $p$ is such that $p(t+T)=$ $-p(t)$ for all $t$. Then equation (1) admits a $2 T$-periodic solution provided (9) is satisfied.

REMARK 5. Condition (9) may seem rather strange compared to the usual existence conditions for harmonics (cf. e.g. [3]), because mostly $|a|$ small causes trouble.

One can easily check that, by (8), (9) (see also the arguments at the beginning of Section 2), Theorem 2 has the following

COROLlary 2. Let the assumptions of Theorem 2 be satisfied. If additionally

$$
|a|<\frac{4}{T(T+2)}-\frac{P+|b|}{\pi},
$$

or

$$
P+|b|<\frac{2 \pi}{T(T+2)} \quad \text { and } \quad a \neq 0,
$$

where $P:=\max _{t \in[0, T]}|p(t)|$, then equation (1) has at least two geometrically distinct $2 T$-periodic solutions.

\section{Concluding remarks (Numerical simulations)}

In spite of the counterexample of R. Ortega [30] (see also his counterargument to the modified Mawhin conjecture in Section 1 of the present paper), the numerical experiments may suggest that in many situations the harmonics or second kind subharmonics, proven to exist for (1) when $a=0$, persist under a small friction. 
Although the bifurcation and state diagrams for (1) are well known (see e.g. [14] and the references therein), we must be very careful, because numerical simulations can very often be misleading as pointed out in [17], [32]. As explained there, the reason is that the conservative or weakly dissipative forced pendula are typical nonhyperbolic systems, and consequently their numerically computed trajectories can be shadowed by the true ones for arbitrarily long times only if special algorithms are applied. As regards equation (1), very precise techniques allowing a high accuracy of the numerically computed orbits have been developed quite recently (see [17], [32]).

\section{REFERENCES}

[1] J. ANDRES, Note to the asymptotic behaviour of solutions of damped pendulum equations under forcing, Nonlinear Anal. 18 (1992), 705-712.

[2] _ Asymptotic properties of solutions to quasi-linear differential systems, J. Comput. Appl. Math. 41 (1992), 57-64.

[3] Several remarks to problem of Moser and conjecture of Mawhin, Boll. Un. Mat. Ital. A (7) 7 (1993), 377-386.

[4] J. Andres, M. Gaudenzi and F. Zanolin, A transformation theorem for periodic solutions of nondissipative systems, Rend. Sem. Mat. Univ. Politec. Torino 48 (1990), 171-186.

[5] J. Andres And S. StanĚK, Note to the Lagrange stability of excited pendulum type equations, Math. Slovaca 43 (1993), 617-630.

[6] S. B. Angenent, The periodic orbits of an area preserving twist map, Comm. Math. Phys. 115 (1988), 353-374.

[7] D. K. Arrowsmith and C. M. Place, Introduction to Dynamical Systems, Clarendon Press, London, 1990.

[8] M. Casdagli, Periodic orbits for dissipative twist maps, Ergodic Theory Dynam. Systems 7 (1987), 165-173.

[9] L Rotational chaos in dissipative systems, Phys. D 29 (1988), 365-386.

[10] A. Chenciner, Sur un énoncé dissipatif du théorème géométrique de Poincaré-Birkhoff, C. R. Acad. Sci. Paris 294 (1982), 243-246.

[11] L. Chiercia ANd E. Zehnder, On asymptotic expansion of quasiperiodic solutions, Ann. Scuola Norm. Sup. Pisa (4) 16 (1989), 254-258.

[12] S.-N. Chow and A. Lasota, On boundary value problems for ordinary differential equations, J. Differential. Equations 14 (1973), 726-737.

[13] L. Collatz, Funktionalanalysis und numerische Mathematik, Springer-Verlag, Berlin, 1964.

[14] D. D'Humieres, M. R. Beasley, B. A. Huberman and A. Libchaber, Chaotic states and routes to chaos in the forced pendulum, Phys. Rev. A 26 (1982), 3483-3496.

[15] A. Fonda, Z. Schneider And F. Zanolin, Periodic oscillations for a nonlinear suspension bridge model, Rech. Math. 18 (1992), Univ. Cath. de Louvain.

[16] J. Franks, Generalizations of the Poincaré-Birkhoff theorem, Ann. of Math. 128 (1988), 139-151.

[17] C. Grebogi, S. M. Hammel, J. A. Yorke and T. Sauer, Shadowing of physical trajectories in chaotic dynamics: Containment and refinement, Phys. Rev. Lett. 65 (1990), 1527-1530. 
[18] C. Grebogi, H. E. Nusse, E. Ott and J. A. Yorke, Basic sets: sets that determine the dimension of basin boundaries, Lecture Notes in Math., vol. 1342, Springer-Verlag, 1988, pp. 220-250.

[19] K. Hocket And P. Holmes, Josephson's junction, annulus maps, Birkhoff attractors, horseshoes and rotation sets, Ergodic Theory Dynam. Systems 6 (1986), 205-239.

[20] P. LE CAlvez, Étude topologique des applications déviant la verticale, Ensaios Mat. 2 (1990), 1-102

[21] Propriétés générales des applications déviant la verticale, Bull. Soc. Math. France 117 (1989), 69-102.

[22] M. Levi, Non-chaotic behavior in the Josephson junction, Phys. Rev. A 27 (1988), 927-931.

[23] Invariant foliations in forced oscillations, Geometry and Analysis in Nonlinear Dynamics, Proc. Workshop Chaotic Dyn. Bifurcations, Groningen, 1989, Pitman Res. Notes Math., vol. 222, Longman Sci.\&Tech., 1992, pp. 34-40.

[24] KAM theory for particles in periodic potentials, Ergodic Theory Dynam. Systems 10 (1990), 777-785.

[25] - Quasiperiodic motions in superquadric time-periodic potentials, Comm. Math. Phys. 143 (1991), 43-83.

[26] J. Mawhin, Nonlinear oscillations: one hundred years after Lyapunov and Poincaré, Z. Angew. Math. Mech. 73 (1993), no. 4-5, 54-62.

[27] J. MAWHin AND M. WILlEM, Multiple solutions of the periodic boundary value problem for some forced pendulum-type equations, J. Differential Equations 52 (1984), 264-287.

[28] M. QIAN, W. X. Shen AND J. Y. Zhang, Global behavior in the dynamical equation of $J-J$ type, J. Differential Equations 71 (1988), 315-333.

[29] J. K. Moser, Quasi-periodic solutions of nonlinear elliptic partial differential equations, Bol. Soc. Brasil. Mat. 20 (1989), 29-45.

[30] R. Ortega, A counterexample for the damped pendulum equations, Bull. Roy. Acad. Sci. Belgique 73 (1987), 405-409.

[31] _ Topological degree and stability of periodic solutions for certain differential equations, J. London Math. Soc. (2) 42 (1990), 505-516.

[32] T. SAUER AND J. A. YORKe, Rigorous verification of trajectories for the computer simulation of dynamical systems, Nonlinearity 4 (1991), 961-979.

[33] G. Tarantello, On the number of solutions for the forced pendulum equation, J. Differential Equations 80 (1989), 79-93.

[34] J. You, Invariant tori and Lagrange stability of pendulum-type equations, J. Differential Equations 85 (1990), 54-65.

[35] Periodic solutions to pendulum type equations, Nanjing Daxue Xuebao Shuxue Bannian Kan 7 (1990), 218-222. (Chinese, English summary)

JAN ANDRES

Department of Mathematical Analysis

Faculty of Science

Palacký University

Tomkova 40

77900 Olomouc-Hejčín, CZECH REPUBLIC

TMNA : Volume $6-1995-\mathrm{N}^{\circ} 1$ 